\title{
Relación del conocimiento táctico de jóvenes futbolistas con la edad, la experiencia y el nivel de pericia
}

\author{
Relationship between young soccer players' tactical \\ knowledge and age, experience and level of expertise
}

\section{Relação entre o conhecimento tático de jovens jogadores sobre a idade, a experiência eo nível de especialização}

\author{
Jaime Serra-Olivares ${ }^{1}$ Luis M. García-López², Antonio Calderón ${ }^{3}$ y Ricardo Cuevas-Campos ${ }^{4}$
}

\begin{abstract}
1 Departamento de Pedagogia en Educación Física. Facultad de Educación. Universidad Católica de Temuco (Chile). 2 Facultad de Educación de Albacete. Universidad de Castilla-La Mancha, Albacete (España). 3 Facultad de Deporte. Universidad Católica de Murcia (España). Guadalupe, Murcia (España) y 4 Facultad de Educación de Ciudad Real. Universidad de Castilla-La Mancha, Ciudad Real (España).
\end{abstract}

Resumen: Dada la importancia del conocimiento previo para decidir de forma adaptable en el deporte, se estudió el conocimiento táctico de 109 jóvenes futbolistas de ocho a doce ańos y su relación con la edad, la experiencia y el nivel de pericia, mediante el Test de Conocimiento Táctico Ofensivo en Fútbol. Se observaron diferencias significativas de magnitud moderada en función de la edad y la experiencia en competición federada de los jugadores $(p<, 05 ; r<, 40)$, aunque los resultados fueron dispares. El nivel de pericia correlacionó significativamente con los conocimientos declarativo $(r h o=, 350 ; p<, 01)$, procedimental $(r h o=, 446 ; p<, 01) \mathrm{y}$ táctico de los jugadores $(r h o=, 446 ; p<, 01)$. Los hallazgos sugieren que la edad $\mathrm{y}$ experiencia no tienen un papel tan determinante sobre el conocimiento táctico como otras variables: metodologías de enseñanza recibidas o la formación del entrenador. Se necesitan estudios en los que se analice si la formación específica en el conocimiento de los problemas tácticos mejora la habilidad de toma de decisiones en el deporte en general y en el fútbol base en particular.

Palabras clave: conocimientos declarativo y procedimental, pericia, edad, experiencia.

Abstract: Given the importance of prior knowledge on adaptable decisionmaking in sport, tactical knowledge of 109 youth soccer players (8-12 years) and its relationship with age, experience and expertise was studied using The Soccer Tactical Knowledge Test. Moderate magnitude significant differences were observed depending on the age and experience in federated competitions of the players $(p<, 05 ; r<, 40)$, although results were mixed. The expertise correlated significantly with declarative $(r h o=, 350 ; p<, 01)$, procedural $(r h o=, 446 ; p<, 01)$ and tactical knowledge of the players ( $r h o$ $=, 446 ; p<, 01)$. Findings suggest that age and experience would not be as decisive over tactical knowledge as other variables not studied: teaching methods, formal instruction, or coach formation. It is necessary more research to examine whether specific formation on tactical problems concepts could improve decision-making in sport and in youth soccer.

Keywords: declarative and procedural knowledge, expertise, age, experience.

Resumo: Dada a importância de uma boa base de conhecimento antes de decidir de forma adaptativa no desporto, reaçôes de 109 jovens futebolistas oito e doze anos e sua relaçáo com o conhecimento, idade, experiência em competição e nível de especializaçáopor meio do Teste ofensivo Futebol Tactical Conhecimento é estudado. Foram observadas diferenças significativas de magnitude moderada versus idade e experiência em competição jogadores federados $(\mathrm{r}<, 40 ; \mathrm{p}<, 05)$, embora os resultados foram mistos. O nível de especializaçáo significativamente correlacionada com o conhecimento declarativo (rho $=350, \mathrm{p}<, 01)$, e os jogadores táticos $(\mathrm{rho}=, 446 ; \mathrm{p}<$ $, 01)$, processual $(\mathrm{rho}=, 446 ; \mathrm{p}<, 01)$. Os resultados sugerem que a idade ea experiência não são decisivo de conhecimento tático como no papel e outras variáveis: metodologias de ensino e formação recebidos do treinador. São necessários mais studos que testam se o treinamento específico no conhecimento dos problemas táticos aumenta a capacidade de tomada de decisáo no desporto em geral eo futebol em particular.

Palavras-chave: declarativo e processual de conhecimento, experiência, idade, experiência.

\section{Introducción}

Las metodologías de enseñanza de los deportes basadas en la comprensión se fundamentan en la idea de que los deportistas toman decisiones de juego a partir de las estructuras de conocimiento previo almacenadas en la memoria, la capaci-

Dirección para correspondencia [Correspondence address]: Jaime SerraOlivares. Departamento de Pedagogía en Educación Física. Facultad de Educación. Universidad Católica de Temuco (Chile). Rudecindo Ortega 02950, Temuco (Chile). E-mail: jserra@uct.cl dad de procesamiento de información, y la interacción de estos limitadores con otros limitadores de la tarea y el contexto (Mitchell, Oslin y Griffin, 2006; Tan, Chow y Davids, 2012). Gracias al contacto con nuevos conceptos y experiencias relacionados con los ya aprendidos, el conocimiento adquiere niveles superiores (Anderson, 1982). Además, al contrastar las primeras concepciones con la realidad, el individuo utiliza la nueva información para construir estructuras más eficientes 
que le permiten decidir adecuadamente al contexto de juego desarrollando patrones motores propios. Por estos motivos se resalta la importancia de la formación-evaluación del conocimiento y la toma de decisiones desde las primeras etapas de iniciación al deporte (Nevett, Rovegno y Baviarz, 2001). No obstante, la influencia de la edad y la experiencia sobre el conocimiento y el proceso decisional sigue siendo una línea de investigación en auge (Fernández-Echeverría et al., 2014; Malina, 2014), y todavía existen dudas sobre qué estructuras cognitivas es necesario desarrollar para la consecución de niveles de pericia superiores (García-López, Contreras Jordán, Penney y Chandler, 2009).

La influencia de la edad y la experiencia sobre el nivel de conocimiento ha sido estudiada con anterioridad en los contextos deportivos escolar (Buscà, Riera y García, 2010; Sánchez-Mora, García-López, Del Valle-Díaz y Martínez-Solera, 2011) y de iniciación (Nevett y French, 1997; McPherson, 1999). Igualmente, la relación entre la edad, la experiencia y el conocimiento ha sido analizada en contextos deportivos específicos de cancha dividida como el tenis (García-González, Moreno-Arroyo, Iglesias, Moreno-Arroyo y Del villar 2006; García-González et al., 2009), o el voleibol (Gil-Arias, Del villar et al., 2012; Gil-Arias, Perla-Moreno et al., 2012; Moreno-Arroyo et al., 2013) y también en otras categorías deportivas como los juegos de invasión como el baloncesto (French y Thomas, 1987) o el fútbol (Kannekens, Elferink-Gemser y Visscher, 2009). Los principales hallazgos indicaron: a) la importancia de la edad para poseer conocimientos superiores y mejor estructurados; b) la influencia de la experiencia en el entrenamiento y competición para representar situaciones de juego de un modo más eficaz; y, c) la necesidad de una buena base de conocimiento sobre la táctica y las reglas de acción para decidir de forma adaptable en el deporte. En definitiva, los resultados han apuntado a una relación directa entre la edad, experiencia, conocimiento específico y pericia.

En el caso concreto del fútbol algunos autores como Kannekens, Elferink-Gemser, Post y Visscher (2009), han evaluado estructuras de conocimiento específico en futbolistas de alto nivel de pericia de 14-18 años. Los hallazgos demuestran un incremento de la habilidad táctica percibida a medida que aumenta la edad. Asimismo, se observaron diferencias de conocimiento en función del rol/posición de los jugadores. Sin embargo, como explicación a estas diferencias los autores no aludieron tanto a la edad como variable determinante, sino más bien la influencia que habría ejercido el proceso de selección y formación de estos jugadores. Se explicaron las diferencias de conocimiento debido a la experiencia, es decir, debido al entrenamiento, la competición y el desarrollo de la pericia, como en estudios anteriores. Este aspecto subraya la necesidad de estudiar en qué estructuras de conocimiento específico están formados los deportistas con talento y qué relación tienen éstas con la pericia. En este sentido, el cono- cimiento específico sobre la táctica ha sido estudiado en jóvenes futbolistas con talento. González-Víllora, García-López, Gutiérrez-Díaz del Campo et al. (2010) y González-Víllora, García-López, Pastor et al. (2011) entre otros, han observado que: a) los jugadores poseen un desconocimiento total de los principios tácticos de juego; b) se aprecian limitaciones en el conocimiento de los elementos técnico-tácticos individuales y grupales; c) los jugadores dominan los conceptos de la técnica pero presentan carencias en el conocimiento de las estrategias-reglas de acción, d) existen limitaciones de conocimiento procedimental, e) existe una relación directa entre el conocimiento y la toma de decisiones.

Tal como se ha justificado en la literatura, los jóvenes que se inician en el deporte han presentado limitaciones en el conocimiento específico de estructuras tan importantes como los problemas tácticos, determinantes de la pericia para tomar decisiones de juego con éxito (Gutiérrez-Díaz del Campo, González-Víllora, y García-López, 2011). Así pues, se intuye que el conocimiento específico de algunas estructuras relacionadas con la táctica (conocimientos declarativo y procedimental sobre la problemática de juego) podrían estar influenciando al desarrollo de la pericia desde las primeras etapas de formación. Por este motivo que el objetivo de este trabajo sea el de analizar la relación entre el nivel de conocimiento táctico de un grupo de jóvenes futbolistas,su edad, experiencia, y nivel de pericia. La hipótesis se inclina porque se observarán diferencias de conocimiento en función de la edad y experiencia de los jugadores. Asimismo, se espera observar una correlación directa entre el nivel de conocimiento táctico y el nivel de pericia de los encuestados.

\section{Método}

\section{Participantes y Contexto}

Se seleccionó un grupo de 109 jóvenes futbolistas de ocho a doce años de alto nivel de pericia pertenecientes a siete equipos en las categorías benjamín y alevín de un club de fútbol profesional. Todos ellos dedicaban al entrenamiento específico de fútbol una media de cinco horas semanales, y poseían entre uno y seis años de experiencia en competiciones federadas en el momento del estudio. Diferían en su nivel de rendimiento de otros jugadores de su misma edad, motivo por el cual fueron considerados como jugadores de alto nivel de pericia (Baker, Cobley, y Schorer, 2012).

\section{Instrumento}

El Test de Conocimiento Táctico Ofensivo en Fútbol (TCTOF) (Serra-Olivares y García-López, en prensa) es un test escrito multi-respuesta con imágenes diseńado para la evaluación de los conocimientos declarativo y procedimental del 
joven futbolista relacionados con la táctica de los juegos de invasión (conocimiento táctico). Mediante esta herramienta se valora el dominio cognitivo del jugador en relación a la problemática contextual táctica o "qué y para qué hacer" durante el juego. Se trata del conjunto de máximas que deben tenerse presentes en función del problema motriz de la situación de juego. Estos problemas son similares en todos los juegos de invasión como el balonmano, baloncesto o el fútbol y se clasifican en problemas tácticos en ataque (conservar la posesión del balón, avanzar hacia la meta contraria y conseguir el objetivo), y en defensa (recuperar el balón, impedir el avance y proteger la propia meta) (Bayer, 1992). En la Tabla 1 se exponen qué variable y dimensiones se evalúan mediante el instrumento, y sus indicadores. Así pues no se trata sim- plemente de la valoración del conocimiento conceptual del jugador, sino que se evalúa también el entendimiento/comprensión del juego dependiendo del contexto táctico de la situación. Este factor permite la evaluación del conocimiento desde una perspectiva más contextualizada, a diferencia de otros instrumentos. En este sentido, el TCTOF consta de dos partes, una escrita para la evaluación del conocimiento declarativo o "saber qué y para qué hacer", como por ejemplo (¿Qué es un pase? o ¿Para qué sirve un pase?), y otra escrita y con figuras para la evaluación del conocimiento procedimental o "saber cómo y cuándo hacer" ante situaciones que siguen el esquema "si... entonces..." (McPherson y Thomas, 1989) (Figura 1).

Tabla 1. Variable, dimensiones e indicadores que se evalúan con el Test de Conocimiento Táctico Ofensivo en Fútbol (Serra-Olivares y García-López, en prensa).

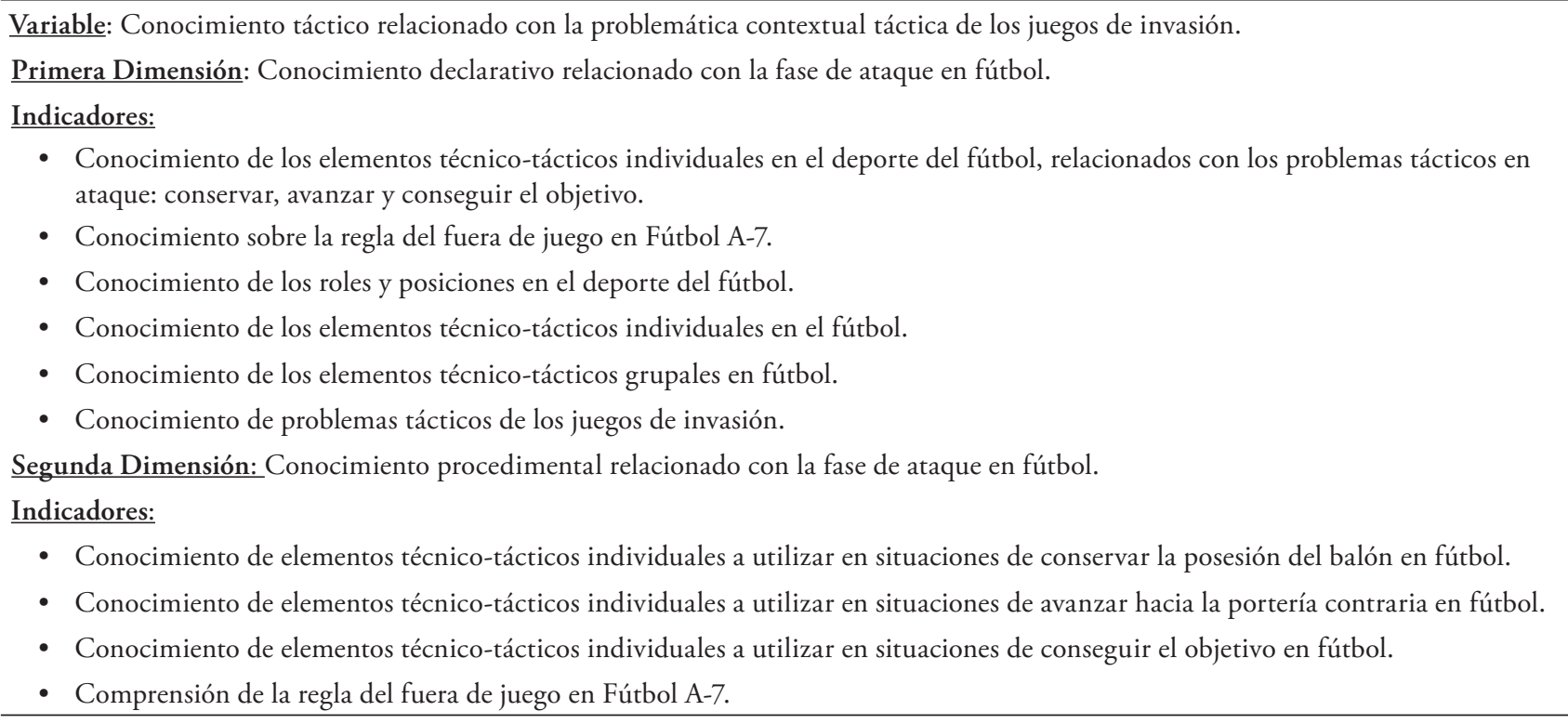
Nota. Cada indicador de la variable es valorado con un número de ítems determinado. 
Figura 1. Ejemplo de ítem de conocimiento procedimental del TCTOF en el que se valora el conocimiento de los elementos técnicotácticos individuales relacionado con los problemas tácticos de juego en ataque.

4. ¿Qué harias si fueras el jugador atacante que lleva el balón?

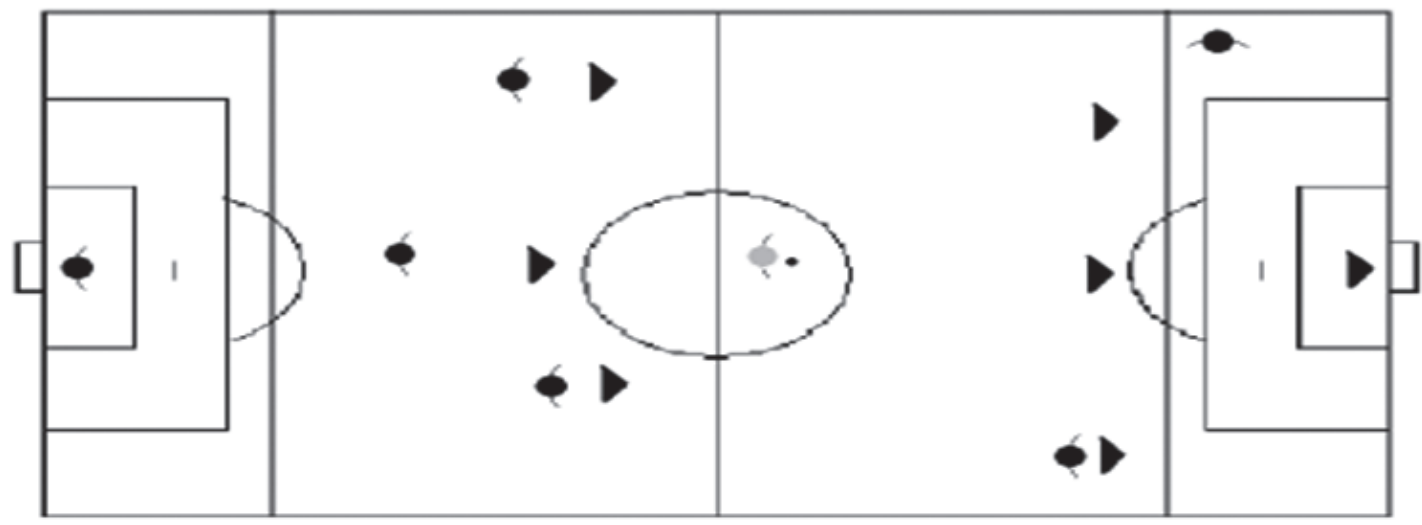

DIRECCIÓN DEL ATAQUE

a) Mantener la posesión del balón y no perderlo.

b) Avanzar hacia la porteria contraria.

c) Intentar marcar un gol.

d) No sabe/ No contesta.

- Realizando para ello:

a) Un pase a un compañero.

b) Una conducción hacia mi porteria, hasta encontrar apoyo de un compañero.

c) Una conducción hacia la porteria contraria, hasta encontrar apoyo de un compañero o tener la posibilidad de tirar.

d) No sabe/No contesta.

El TCTOF es un instrumento que ha sido validado previamente (Serra-Olivares y García-López, en prensa). Presenta resultados adecuados respecto a la validez de contenido (acuerdo de expertos en adecuación, inteligibilidad y representatividad de los ítems), estabilidad ( $r h o=, 75 ; p<, 01)$, y correlación ítem-total corregida $(\alpha>, 87)$. En todo caso, se calculó la fiabilidad del TCTOF con los participantes de esta investigación observándose valores de fiabilidad de $\alpha>, 87$ para todas las variables, dimensiones e indicadores del instrumento.

\section{Procedimiento}

Se realizó un diseño comparativo, transversal y correlacional (Thomas y Nelson, 2007). Se obtuvo el consentimiento informado por parte de los padres de los jugadores encuestados para el desarrollo del estudio de acuerdo a las directrices éticas de la Declaración de Helsinki de 1975, revisada en 2000. Posteriormente, se contactó con los profesionales del club encargados del entrenamiento y formación de los jugadores participantes para fijar las fechas de realización de cada una de las dos partes del TCTOF. Así, después de establecer los días de entrenamiento de los equipos benjamín y alevín como el mejor momento para pasar los tests, se seleccionó la sala de prensa del club para su realización. Coincidiendo con el tramo final de la temporada para todos los jugadores, se realizaron las dos partes del tests en cuatro días diferentes de las dos últimas semanas del mes de abril de 2012. Primero, se realizó la evaluación del conocimiento táctico (declarativo y procedimental) a todos los jugadores en categoría benjamín en dos lunes consecutivos, y posteriormente, en dos miércoles consecutivos a los jugadores en categoría alevín. La duración de estas sesiones fue de quince minutos aproximadamente cada día. Cabe decir que previa a la realización de cada una de las dos partes del test el investigador principal realizaba una pequeña introducción, y quedaba perfectamente claro el modo en el que se debía contestar a cada parte del test. Además, quedaba registrado: a) el nombre y apellidos del jugador encuestado, b) la edad cronológica en años, c) la categoría de competición en fútbol en la que se encontraba jugando, y d) la experiencia en ańos en competiciones de fútbol similares a la que se encontraba en ese momento. Paralelamente, se solicitó 
al entrenador de cada equipo que valorase el nivel de pericia de sus jugadores, como en otros trabajos (Buscà et al., 2010). Siguiendo las instrucciones del investigador principal, cada entrenador puntuó a sus jugadores de 0 a 10 (considerando que $10=$ mejor/mejores jugadores dentro de la categoría en la que competía el jugador, 0 = jugador/es con el nivel más bajo).

\section{Análisis de datos}

Se realizó el cálculo de normalidad y homocedasticidad de las variables mediante las pruebas de Kolgomorov-Smirnov y de Levene. Estas indicaron que no todas las variables cumplían el supuesto de normalidad. Por este motivo se realizó el análisis inferencial mediante las pruebas no paramétricas de UMann Whitney para analizar las diferencias de conocimiento de los jugadores en función de su edad y experiencia en competición federada, y el análisis correlacional mediante la prueba Rho Spearman, para determinar la relación existente entre el conocimiento de los jugadores y su nivel de pericia (valorado por los entrenadores de los jugadores encuestados). Para ello, se clasificó a los participantes en cinco grupos de edad (ocho, nueve, diez, once y doce años), y en siete grupos de experiencia en competición federada (cero, uno, dos, tres, cuatro, cinco y seis años). Se consideró el nivel de significatividad de las diferencias de $(p<, 05)$ para todo el análisis. El cálculo del tamaño del efecto de las diferencias se realizó con la fórmula $(r=Z / \sqrt{ } N)$, donde $N$ era el número total de participantes en cada caso. Finalmente, se clasificó la magnitud de las diferencias significativas en diferencias pequeñas $(r=, 10)$, moderadas $(r=, 30)$ y grandes $(r=, 50)$, dado que en este tipo de estudios suelen ser hallados tamańos del efecto menores que en otros campos (Morales, 2011). Cabe destacar que el cálculo de la magnitud del efecto se considera un análisis de gran potencial para evaluar en qué medida las diferencias observadas se pueden atribuir a las variables analizadas (Baguley, 2009). Por este motivo, que fuese calculado en este trabajo.

\section{Resultados}

Diferencias de conocimiento en función de la edad de los participantes

No se observaron diferencias significativas de conocimiento entre los grupos de edad de ocho y nueve años en ninguna de las dimensiones del instrumento. Sin embargo, el grupo de diez años presentó unos conocimientos procedimental $(U$ $=14.500 ; p<, 05 ; r=-, 30)$ y táctico $(U=12.500 ; p<, 05$; $r=-, 31)$ significativamente superiores a sus compañeros de ocho años, aunque estas diferencias no fueron significativas en el conocimiento declarativo. Igualmente, el grupo de 11 ańos de edad presentó unos conocimientos declarativo $(U=$ 27.500; $p<, 05 ; r=-.31)$, procedimental $(U=14.000 ; p<, 05$; $r=-, 38)$ y táctico $(U=12.500 ; p<, 05 ; r=-, 38)$ significativamente superiores al grupo de ocho ańos. Estas diferencias también fueron halladas al comparar el grupo de ocho con el de doce años de edad, observándose diferencias significativas a favor del grupo de mayor edad en el conocimiento declarativo $(U=10.000 ; p<, 05 ; r=-, 28)$, el procedimental $(U=$ 8.000; $p<, 05 ; r=-, 30)$, y el táctico $(U=5.500 ; p<, 05 ; r=$ $-, 33)$. No se observaron diferencias significativas en ninguna de las dimensiones del instrumento al comparar los grupos de nueve y diez años de edad. Las diferencias sí fueron significativas entre los grupos de nueve y once ańos. En este sentido, los jugadores de mayor edad presentaron unos conocimientos declarativo $(U=185.000 ; p<, 05 ; r=-24)$, procedimental $(U=183.500 ; p<, 05 ; r=-, 25)$ y táctico $(U=168.500 ; p<$ ,05; $r=-, 28)$, significativamente superiores a los jugadores más jóvenes. Las diferencias también fueron significativas al comparar los grupos de nueve y doce años de edad. De nuevo, el grupo de mayor edad presentó unos conocimientos declarativo $(U=62.500 ; p<, 05 ; r=-, 24)$ y táctico $(U=40.500 ; p$ $<, 05 ; r=-, 33)$ significativamente superiores, no así en el conocimiento procedimental. Las diferencias tampoco fueron significativas al comparar los grupos de edad de diez y once años. Sin embargo, las diferencias de conocimiento sí fueron significativas al comparar los grupos de diez y doce años de edad. De nuevo, el grupo de mayor edad presentó unos conocimientos procedimental $(U=28.500 ; p<, 05 ; r=-, 35)$ y táctico $(U=28.500 ; p<, 05 ; r=-, 30)$ significativamente superiores, no así en el conocimiento declarativo. Finalmente, no se observaron diferencias significativas entre los grupos de once y doce años.

Diferencias de conocimiento en función de la experiencia de los participantes en competición federada

No se observaron diferencias significativas de conocimiento al comparar los grupos de jugadores de cero y un ańo de experiencia en competición federada. Las diferencias tampoco fueron significativas al comparar el grupo de cero con los grupos de dos, tres, cuatro y cinco años de experiencia. Las diferencias fueron significativas al comparar el grupo de cero con el de cinco ańos de experiencia. En este sentido los jugadores con mayor experiencia mostraron un conocimiento declarativo significativamente superior $(U=8.000 ; p<, 05 ; r=-, 24)$. No se hallaron diferencias significativas de conocimiento al comparar el grupo de 1 ańo con los grupos de dos, tres y cuatro años de experiencia. Las diferencias fueron significativas al comparar el grupo de uno con el de cinco años. En este caso el grupo de mayor experiencia presentó un conocimiento procedimental significativamente superior $(U=6.000 ; p<$ $, 05 ; r=-, 22)$. Igualmente, el grupo de seis años de experiencia presentó un conocimiento declarativo significativamente superior al grupo de 1 año $(U=3.000 ; p<, 05 ; r=-, 21)$. Al 
comparar los grupos de dos y tres años se observó que los jugadores con mayor experiencia poseían un conocimiento táctico significativamente más alto que sus compañeros $(U=$ $88.000 ; p<, 05 ; r=-, 23)$, aunque las diferencias no fueron significativas el comparar el nivel de conocimientos declarativo y procedimental por separado. No se observaron diferencias al comparar los grupos de dos y cuatro ańos de experiencia. Las diferencias fueron significativas al comparar los grupos de dos y cinco años, observándose unos conocimientos declarativo $(U=58.500 ; p<, 05 ; r=-, 26)$, procedimental $(U$ $=47.500 ; p<, 05 ; r=-, 32)$ y táctico significativamente superiores de los jugadores con mayor experiencia $(U=48.000 ; p$ $<, 05 ; r=-, 31)$. Las diferencias también fueron significativas a favor del grupo de mayor experiencia al comparar los grupos de dos y seis años, tanto en el conocimiento táctico $(U=$ 21.000; $p<, 05 ; r=-, 31)$, como en el declarativo $(U=16.000$; $p<, 05 ; r=-, 35)$. No se observaron diferencias significativas entre los grupos de tres y cuatro ańos de experiencia. Estas tampoco fueron significativas al comparar el grupo de tres y cinco años. Las diferencias fueron significativas a favor del grupo de mayor experiencia al comparar los grupos de tres y seis ańos en el conocimiento declarativo $(U=24.500 ; p<, 05$; $r=-22)$, no así en los conocimientos procedimental y táctico. Se observó un conocimiento procedimental significativamente superior del grupo de cinco años respecto al de 4 años de experiencia $(U=60.500 ; p<, 05 ; r=-, 25)$, aunque estas no fueron significativas en los conocimientos declarativo y táctico. Las diferencias sí fueron significativas a favor del grupo de mayor experiencia al comparar los grupos de cuatro y seis años, tanto en el conocimiento declarativo $(U=23.000 ; p$ $<, 05 ; r=-, 30)$, como en el táctico $(U=31.000 ; p<, 05 ; r=$ -,26), no así en el procedimental. No se observaron diferencias significativas al comparar los grupos de cinco y seis años de experiencia.

Relación entre el nivel de pericia de los jugadores y el tipo de conocimiento evaluado

Independientemente de la edad de los jugadores, los resultados muestran una relación significativa, proporcional y directa entre el nivel de pericia de los jugadores participantes y su nivel de conocimientos declarativo, procedimental y táctico..

Tabla 2. Relación entre el nivel de pericia de los jugadores y el tipo de conocimiento, independientemente de la edad.

\begin{tabular}{lcc}
\hline Tipo de conocimiento & $\begin{array}{c}\text { Correlación } \\
\text { Rho Spearman }\end{array}$ & Significación \\
\hline Conocimiento declarativo &, $350^{* *}$ &, 001 \\
Conocimiento procedimental &, $446^{* *}$ &, 000 \\
Conocimiento táctico &, $466^{* *}$ &, 000 \\
\hline
\end{tabular}

**La correlación es significativa a nivel de 0,01 (bilateral).

\section{Discusión}

El objetivo del presente trabajo fue analizar el nivel de conocimientos declarativo, procedimental y táctico de un grupo de jóvenes futbolistas, y su relación con la edad, la experiencia en competición y el nivel de pericia. Los hallazgos indican que la edad, en mayor medida que la experiencia en competición federada fue determinante sobre el nivel de conocimientos de los participantes, aunque los resultados fueron dispares. Por otro lado, se observa una relación directa entre el conocimiento y el nivel de pericia. Los resultados demostraron que los jugadores de mayor edad poseían unos conocimientos significativamente superiores a sus compañeros más jóvenes, al igual que en otros estudios (Moreno-Arroyo et al., 2013). Sin embargo, las diferencias no fueron significativas al realizar los pares de comparaciones de los grupos de ocho y nueve ańos, los grupos de nueve y diez ańos y los grupos de once y doce años de edad, señalando la necesidad de poseer al menos dos años de diferencia cronológica para disfrutar de un mayor conocimiento táctico a estas edades. Esta idea ha sido sugerida por Gutiérrez-Díaz del Campo et al. (2011) al analizar la adaptación a los problemas tácticos de iniciados de la misma edad en el contexto escolar, y subraya la importancia del crecimiento, la maduración y el desarrollo conductual para la formación de estructuras cognitivas pertinentes en el desarrollo del conocimiento táctico y la toma de decisiones (Malina, 2014). Por otro lado, no se observaron diferencias en algunas parcelas de conocimiento en función de la edad en este trabajo, como por ejemplo al comparar el conocimiento declarativo entre los grupos de ocho y diez y de diez y doce ańos, o el conocimiento procedimental entre los grupos de diez y doce años. De este modo, las diferencias de conocimiento observadas podrían deberse a factores no controlados en este trabajo como son: las metodologías de enseńanza recibidas por los jugadores, como se observó en otros trabajos (Kannekens et al., 2009; Nevett et al., 2001; Sánchez-Mora et al., 2011), la capacidad cognitiva, como limitador del individuo (Tan et al., 2012), o el nivel de formación de los entrenadores encargados del entrenamiento de los jugadores encuestados. En este sentido, no podemos olvidar que la integración de competencias como la intelectual, la motriz o la cognitiva, involucran la interacción de distintos procesos biológicos como el crecimiento y la maduración, y de otros procesos conductuales como el desarrollo, propios de la socialización cultural. Este hecho resalta la necesidad de realizar una aproximación biocultural a la formación del conocimiento y la toma de decisiones en el deporte (Malina, 2014).

En el caso de la experiencia se aprecian hallazgos similares. A pesar de que se observaron diferencias significativas de conocimiento a favor de los jugadores de mayor experiencia en competición federada en varios casos (por ejemplo entre el grupo de cinco años con los de cero, uno, dos y cuatro de 
experiencia, o entre el grupo de seis y los de uno, dos, tres y cuatro), estas fueron muy dispares. Si bien es cierto que la experiencia en competición se ha mostrado como un factor determinante del conocimiento en otros estudios (French y Thomas, 1987; García-Gonzálezet al., 2009; Gil-Arias, DelVillar et al., 2012; Nevett y French, 1997), los hallazgos de este trabajo sugieren que la experiencia no habría sido un factor tan influyente como otras variables no controladas que se consideran fundamentales para el aprendizaje como: el aprendizaje social, los métodos utilizados o la formación del entrenador (Mitchell et al., 2006; Tan et al., 2012).

De modo que los jóvenes futbolistas de este trabajo no necesariamente estarían acumulando un mayor conocimiento sobre la funcionalidad de los problemas tácticos, simplemente por aumentar la cantidad de experiencia en una serie de competiciones futbolísticas. Por el contrario, se intuye que el conocimiento de estos deportistas será mayor si los estímulos que rodean su aprendizaje facilitan realmente la organización de los conceptos ya aprendidos, y la estructuración de los nuevos conocimientos (Nevett et al., 2001). Esta cuestión es de gran importancia cuando se trata de la formación de jóvenes que se inician al deporte, en la que el tipo de metodología recibida y los conocimientos adquiridos poseen un papel fundamental sobre las habilidades de toma de decisión (Alarcón et al., 2009; García-López et al., 2009; Tan et al., 2012). Esta sugerencia se ve apoyada también por los resultados observados en este trabajo en relación al conocimiento táctico y la pericia de los jugadores, que mostraron una relación directa y proporcional entre las dos variables, como en otros estudios (Baker et al., 2012; García-González et al., 2009).

Por tanto, teniendo en cuenta la influencia que podrían ejercer los métodos de enseńanza sobre el desarrollo del conocimiento, y asimismo la influencia del conocimiento sobre la pericia, cabe preguntarse cómo se debería organizar el proceso de enseñanza de los deportes como el fútbol para favorecer la consecución de altos niveles de rendimiento. En este sentido, los modelos comprensivos de enseñanza de los deportes resaltan la importancia de ciertos factores (lenguaje específico, andamiaje de conocimientos, aprendizaje social), como factores determinantes a la hora de fomentar la apreciación del juego y la conciencia táctica (Mitchell et al., 2006), no existe una planificación justificada de los contenidos conceptuales a tratar en iniciación deportiva. En este sentido, esta investigación aporta una visión más al estudio del conocimiento en el deporte, analizando el dominio cognitivo de la problemática táctica de los juegos de invasión como el fútbol. Se trata de estructuras fundamentales en las que los jóvenes han demostrado poseer serias carencias y que al parecer, podrían ayudar al desarrollo de la pericia, como sugieren los resultados de este y otros trabajos de investigación (González-Víllora, García-López, Gutiérrez-Díaz del Campo et al., 2010; GonzálezVíllora, García-López, Pastor et al., 2010).

\section{Aplicaciones prácticas}

A pesar de poseer un alto control de las habilidades de toma de decisión que permiten desarrollar niveles de rendimiento deportivo más alto, los jugadores participantes en esta investigación demostraron un conocimiento muy básico sobre la problemática contextual y la táctica de los juegos de invasión como el fútbol. Este hecho, unido a la relación observada entre el conocimiento táctico y la pericia, y a que ni la edad ni la experiencia fueron la causa principal de las diferencias de conocimiento halladas, justifica la siguiente afirmación. Son necesarios más estudios en los que se analice el conocimiento de los problemas tácticos de los juegos de invasión y su relación con la pericia, y en los que se preste mayor atención al tipo de metodología de enseñanza recibida por los jugadores ¿Podría la formación teórica en la problemática táctica de juego contribuir a la consecución de niveles de rendimiento superiores?

Este enfoque de análisis permitiría profundizar sobre aquellos dominios de conocimiento en los cuáles los iniciados poseen mayores carencias, y supone un paso más hacia el diseńo de programas y herramientas de enseńanza-aprendizaje de los deportes de calidad. En este sentido, el instrumento de evaluación del conocimiento táctico utilizado en este estudio se presume como una herramienta útil, de fácil empleo y económica para la evaluación del aprendizaje futbolístico desde la perspectiva de la pedagogía no lineal. La realización de evaluaciones periódicas sobre el conocimiento de la táctica deportiva a los iniciados, contribuiría a la adecuación de los contenidos a desarrollar a las necesidades específicas de los mismos. Además, teniendo en cuenta la relación existente entre el nivel de conocimiento y pericia, la información relativa a evaluaciones similares podría ser utilizada para clasificar a los jugadores por grupos de nivel, detectar a aquellos que poseen mayores limitaciones y, distinguir a aquellos otros que poseen unos conocimientos superiores. Por el contrario, sin este tipo de información es posible que no se atienda correctamente a los jugadores con mayores carencias de conocimiento, y muy probable, que aquellos otros jugadores que no están recibiendo los estímulos necesarios, estén limitando su potencial.

Con instrumentos de estas características ha sido posible demostrar, por ejemplo, que existe una fuerte relación entre el conocimiento sobre la táctica deportiva y el nivel de pericia de los jugadores, o que existen franjas de tiempo necesarias para alcanzar ciertos niveles de entendimiento del juego. Por todo lo anterior, se sugiere la necesidad de continuar esta línea de estudio, concentrando la atención en la comprensión del conocimiento sobre la táctica deportiva y su relación con la pericia, como medio de mejorar el rendimiento deportivo. Para ello será necesaria la búsqueda de aquellos métodos de enseñanza más eficientes para alcanzar niveles superiores, y un ajuste de los contenidos enseńados a dicho nivel de pericia. 


\section{Referencias}

1. Alarcón, F., Cárdenas, D., Miranda, M. T., Ureña, N., Piñar, M. I., y Torre, E. (2009). Effect of a training program on the improvement of basketball players' decision making. Revista de Psicología del Deporte, 18, 403-407.

2. Anderson, J. R. (1982). Acquisition of cognitive skill. Psychological Review, 89, 369-406.

3. Baker, J., Cobley, S., y Schorer, J. (2012). Talent identification and development in Sport. International Perspectives. London: Routledge.

4. Bayer, C. (1992). La enseñanza de los juegos deportivos colectivos. Barcelona: Hispano Europea.

5. Baguley, T. (2009). Standardized or simple effect size: what should be reported? British Journal of Psychology, 100(3), 603-17.

6. Buscà, B., Riera, J., y Garcia, L. (2010). Diseño de un nuevo test para evaluar las aptitudes cognitivas en el deporte. Estudio de fiabilidad y validez. Revista de Psicología del deporte, 19(2), 277-290.

7. Fernández-Echeverría, C., Moreno-Arroyo, A., Gil-Arias, A., ClaverRabaz, F., Moreno-Arroyo, P. (2014). Estudio del conocimiento procedimental, experiencia y rendimiento, en jóvenes jugadores de voleibol. Retos. Nuevas tendencias en Educación Física, Deporte y Recreación, 25 13-16.

8. French, K. E., y Thomas, J. R. (1987). The relation of knowledge development to children's basketball performance. Journal of Sport Psychology, 9, 15-32.

9. García-González, L., Moreno-Arroyo, M. P., Iglesias, D., MorenoArrollo, A., y Del villar, F. (2006). El conocimiento táctico en tenis. Un estudio con jugadores expertos y noveles. Cuadernos de Psicología del Deporte, 6(2), 11-20.

10. García-González, L., Moreno-Arroyo, M. P., Moreno-Arroyo, A., Iglesias, D., y Del villar, F. (2009). Estudio de la relación entre conocimiento y toma de decisiones en jugadores de tenis, y su influencia en la pericia deportiva. Revista internacional de ciencias del deporte, 17, 65-75.

11. García-López, L. M., Contreras Jordán, O. R., Penney, D., y Chandler, T. J. L. (2009). The role of transfer in games teaching: Implications in the development of the sports curriculum. European Physical Education Review, 15(1), 47-63.

12. Gil-Arias, A., Del villar, F., Claver, F., Moreno-Arroyo, A., GarcíaGonzález, L., y Perla-Moreno, M. (2012). ¿Existe relación entre el nivel de competición y el conocimiento del juego en voleibol?. Retos. Nuevas tendencias en Educación Física, Deporte y Recreación, 21, 53-57.

13. Gil-Arias, A., Perla-Moreno, M., García-González, L., Moreno-Arroyo, A., y Del villar, F (2012). Analysis of declarative and procedural knowledge in volleyball according to the level of practice and players' age. Perceptual \& Motor Skills: Exercise \& Sport, 115(2), 632-644.

14. González-Víllora, S., García-López, L. M., Contreras-Jordán, O. R., y Gutiérrez-Díaz del Campo, D. (2010). Estudio descriptivo sobre el desarrollo táctico y la toma de decisiones en jóvenes jugadores de fútbol (12 años). Revista Infancia y Aprendizaje, 33(4), 489-501.

15. González-Víllora, S., García-López, L. M., Pastor-Vicedo, J. C., y Contreras-Jordán, O. R. (2011). Conocimiento táctico y toma de decisiones en jóvenes jugadores de fútbol (10 años). Revista de Psicología del Deporte, 20(1), 79-97.

16. Gutiérrez-Díaz del Campo, D., González-Víllora, S., y García-López
(2011). Differences in decision-making development between expert and novice invasion game players. Perceptual and Motor Skills, 112(3), 871-888. doi: 10.2466/05.10.11.25

17. Kannekens, R., Elferink-Gemser, M. T., Post, W. J., y Visscher, C. (2009). Self-assessed tactical skills in elite youth soccer players: a longitudinal study. Perceptual and Motor Skills, 109(2), 459-472. doi: 10.2466/pms.109.2.459-472.

18. Kannekens, R., Elferink-Gemser, M. T. y Visscher, C. (2009). Tactical skills of world-class youth soccer teams. Journal of Sports Sciences, 27(8), 807-812. doi: 10.1080/02640410902894339.

19. Malina, R. M. (2014). Top 10 research questions related to growth and maturation of relevance to physical activity, performance, and fitness. Research Quarterly for Exercise and Sport, 85(2), 157-173. doi:10.1080/ 02701367.2014.897592.

20. McPherson, S. L., y Thomas, J. R. (1989). Relation of knowledge and performance in boys' tennis: age and expertise. Journal of Experimental Child Psychology, 48, 190-211.

21. McPherson, S. L. (1999). Expert-novice differences in performance skills and problem representations of youth and adults during tennis competition. Research Quarterly for Exercise and Sport, 70(3), 233-251.

22. Mitchell, S. A., Oslin, J. L., y Griffin, L. (2006). Teaching sport concepts and skills. A tactical approach. Champaign, IL: Human Kinetics.

23. Morales, P. (2011). Guía para construir cuestionarios y escalas de actitudes. Guatemala: Universidad Rafael Landívar. (Disponible en http:// www.upcomillas.es/personal/peter/otrosdocumentos/Guiaparaconstruirescalasdeactitudes.pdf).

24. Moreno-Arroyo, A., Del villar, F., García-González, L., García-Calvo, T., y Moreno, M. P. (2013). Propiedades psicométricas de un cuestionario para la evaluación del conocimiento procedimental en voleibol (CCPV). RICYDE, 30(9), 38-47. http://dx.doi.org/10.5232/ ricyde2013.03103.

25. Nevett, M. E., y French, K. E. (1997). The development of sport-specific planning, rehearsal, and updating of plans during defensive youth baseball game performance. Research Quarterly for Exercise and Sport, 68, 203-214.

26. Nevett, M., Rovegno, I., y Baviarz, M. (2001). Chapter 8. Fourthgrade children's knowledge of cutting, pass and tactics in invasion games after a 12-lesson units of instruction. Journal of teaching in physical education, 20, 389-401.

27. Sánchez-Mora, D., García-López, L. M., Del Valle-Díaz, M.S., y Martínez-Solera, I. (2011). Spanish primary school students' knowledge of invasion games. Physical Education \& Sport Pedagogy, 16(3), 251-264.

28. Serra-Olivares, J., y García-López, L. M. (201x). Diseño y validación del test de conocimiento táctico ofensivo en fútbol (TCTOF). Revista Internacional de Medicina y Ciencias de la Actividad Física y el Deporte (en prensa).

29. Tan, C. W. K, Chow, J. I., y Davids, K. (2012). 'How does TGfU work?': examining the relationship between learning design in TGfU and a nonlinear pedagogy. Physical Education and Sport Pedagogy, $17(4), 331-348$.

30. Thomas, J. R. y Nelson, J. K. (2007). Métodos de investigación en actividad física. Badalona: Paidotribo. 